\title{
Layer dependent neural modulation of a realistic layered-microcircuit model in visual cortex based on bottom-up and top-down signals
}

\author{
Nobuhiko Wagatsuma ${ }^{1,2^{*}}$, Tobias C Potjans ${ }^{3,4,5}$, Markus Diesmann ${ }^{1,4}$, Tomoki Fukai ${ }^{1,4,6}$ \\ From Twentieth Annual Computational Neuroscience Meeting: CNS*2011 \\ Stockholm, Sweden. 23-28 July 2011
}

Visual attention allocates the information processing resources of the brain to selected sensory inputs to reduce the processing load. Here, we carried out a large-scale simulation with a layered-microcircuit model of the visual cortex based on current knowledge of cortical neurobiology to explore the complex interaction between bottom-up visual input and top-down attentional signals. The microcircuit model corresponds to two columns of visual cortex. Each column consists of about 40,000 integrate-and-fire neurons comprising layers $2 / 3,4,5$ and 6 [1]. These columns interact via lateral inhibition from layer $2 / 3$ excitatory neurons in one column to layer $2 / 3$ inhibitory neurons in the other, so that the full network comprises in total around 80,000 neurons and 300 million synapses. Excitatory and inhibitory neurons in layer 4 of the model receive visual inputs mimicking vertical and horizontal bars. Topdown attention was projected to layers $2 / 3$ and 5 to facilitate the visual processing. In order to investigate the mechanism of visual processing and the attentional effect in the layered-microcircuit model, we compared the response of the model to physiological results for neural and attentional modulation [2]. Our model successfully reproduced previous experimental observations of stimulus presentation and attentional effects on visual neuronal responses in layers $2 / 3$ and 5 (Figure 1). Moreover, the model predicts contrasting differences in the effects of attention between cortical layers: attention to a preferred stimulus of a column enhances neuronal responses of layers $2 / 3$ and 5 , the output station of cortical microcircuits, whereas attention suppresses neuronal activities of layer 4, the input station of cortical

\footnotetext{
* Correspondence: nwagatsuma@brain.riken.jp

'RIKEN Brain Science Institute, 2-1 Hirosawa, Wako, Saitama 351-0198, Japan Full list of author information is available at the end of the article
}

microcircuits. Further simulations with our model suggest that the specific attentional modulation pattern of layer 4 activity emerges from inter-laminar synaptic connections within the cortical microcircuit and is crucial

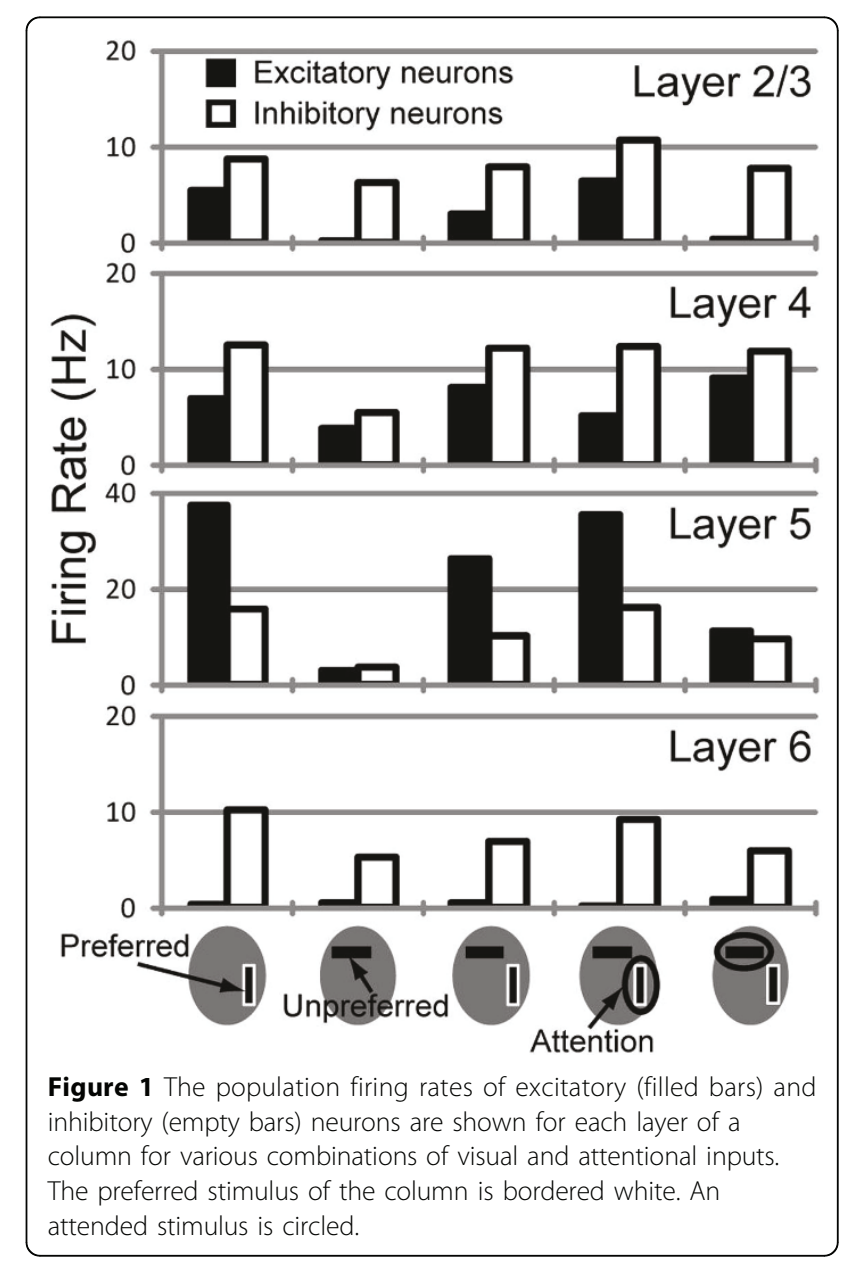


for the microcircuit to rapidly shift attention to a stimulus different from the one currently attended to. Our multicolumnar model is a canonical microcircuit model for the dynamic selection of multiple sensory inputs to the visual cortices, and makes several testable predictions about the layer-dependence of the response modulations.

\section{Acknowledgements}

This work was partly supported by Grants-in-Aid for Scientific Research on Innovative Areas (no. 22115013), the Next-Generation Supercomputer Project of MEXT, Japan, and EU Grant 15879 (FACETS) and the Helmholtz Alliance on Systems Biology.

\section{Author details}

RIKEN Brain Science Institute, 2-1 Hirosawa, Wako, Saitama 351-0198, Japan. ${ }^{2}$ Japan Society for the Promotion of Science, Japan. ${ }^{3}$ Institute of

Neuroscience and Medicine, Computational and Systems Neuroscience (INM6), Research Center Juelich, Juelich, Germany. ${ }^{4}$ Brain and Neural Systems Team, RIKEN Computational Science Research Program, 2-1 Hirosawa, Wako, Saitama 351-0198, Japan. ${ }^{5}$ Faculty of Biology III, Albert-Ludwigs-University Freiburg, Schaenzlestrasse 1, 79104 Freiburg, Germany. ${ }^{6}$ CREST, JST, Honmachi, 4-1-8, Kawaguchi, Saitama, Japan.

Published: 18 July 2011

\section{References}

1. Potjans TC, Diesmann M: The cell-type specific connectivity of the local cortical network explains prominent features of neuronal activity., submitted.

2. Reynolds JH, Chelazzi L, Desimone R: Competitive mechanisms subserve attention in macaque areas V2 and V4. J Neurosci 1999, 19:1736-1753.

doi:10.1186/1471-2202-12-S1-P114

Cite this article as: Wagatsuma et al:: Layer dependent neural modulation of a realistic layered-microcircuit model in visual cortex based on bottom-up and top-down signals. BMC Neuroscience 201112 (Suppl 1):P114.

\section{Submit your next manuscript to BioMed Central and take full advantage of:}

- Convenient online submission

- Thorough peer review

- No space constraints or color figure charges

- Immediate publication on acceptance

- Inclusion in PubMed, CAS, Scopus and Google Scholar

- Research which is freely available for redistribution

Submit your manuscript at www.biomedcentral.com/submit
(Ciomed Central 